\title{
Developing therapeutic monoclonal antibodies at pandemic pace
}

\author{
The time from discovery to proof-of-concept trials could be reduced to 5-6 months from a traditional timeline \\ of 10-12 months.
}

Brian Kelley

Com utbreaks of emerging infectious diseases have become increasingly common in recent decades.

Epidemics have spread across the globe, including AIDS, H1N1 influenza and most recently coronavirus disease (COVID-19). In the face of a pandemic infectious disease outbreak, new approaches should be explored to enable the most rapid evaluation of antibodies for passive immunization or treatment. The fastest timeline from discovery to clinical evaluation of novel recombinant antibodies for medical use has been a focus of the biopharmaceutical industry for decades. For potentially life-saving therapies, the benefits of the earliest clinic testing should translate to accelerated pivotal trial testing and maximal patient benefit. Process and product development groups at major biopharmaceutical companies have reduced phase 1 timelines for recombinant antibody production through a universal convergence on similar technologies and strategies. Yet there may be opportunities for substantially faster timelines arising from a combination of the latest technological advances with acceptance of higher business risk or costs without an increased risk profile to patients in the first clinical trials.

\section{A faster path}

During a pandemic, there is no time to waste in the development and clinical testing of therapeutic modalities, including vaccines, nucleic acids, small molecules, convalescent serum, intravenous immunoglobulin G (IgG) and monoclonal antibodies (mAbs).

Until recently, the evaluation of $\mathrm{mAb}$ therapies for this scenario has been slow and the production capacity was limited. What has changed to enable rapid evaluation of $\mathrm{mAb}$ therapies in this case?

The product development timeline from lead $\mathrm{mAb}$ identification to phase 1 investigational new drug application (IND) is $10-12$ months at many companies today - a dramatic reduction from the 18 months that was standard in the industry 5 or more years ago. A combination of recent technical advances and the acceptance of business (but not product quality or patient safety) risks offers a further acceleration for clinical trials. Rapid clinical production capacity has benefited from development of highly productive cell lines and larger bioreactors using single-use technology, enabling the production of thousands of doses from a single batch of over 5 kilograms.

Today, we can accelerate these activities and enable production capacity for clinical studies for therapeutic mAbs. What could be the fastest path to provide mAbs for clinical evaluation during a pandemic outbreak? I propose that the answer could be 5-6 months, rather than 10-12 months.

\section{Lead $\mathrm{mAb}$ identification and characteristics}

In a conventional discovery program, $\mathrm{mAb}$ identification usually takes several months to identify an attractive candidate. But one way of increasing the speed of identifying leads is to screen prospectively isolated panels against new pathogens and many viral strains ${ }^{1-3}$. This enables rapid identification of the best $\mathrm{mAb}$ for development and puts process development and manufacturing on the critical path to clinical evaluation. There are several other $\mathrm{mAb}$ discovery approaches (reviewed in ref. ${ }^{4}$ ) that may also be capable of rapid lead identification that would subsequently benefit from this development strategy.

I present here an assessment of accelerated $\mathrm{mAb}$ discovery and development based on the use of the clinically proven IgG1 isotype. Over 50 IgG1 therapeutic mAbs have been commercialized ${ }^{5}$ and hundreds more have been clinically tested. The IgG1 mAb safety and quality risk profiles are low and enable a shift in risk tolerance. Substantial platform knowledge, product development history, current good manufacturing practice (cGMP) production experience and facilities are also broadly established for IgG1 mAbs. In response to a pandemic disease outbreak, IgG1 mAbs therefore have a distinct advantage as prophylactic or therapeutic biological agents.

\section{Speed to phase 1 cell line}

To accelerate clinical development, the production host should be a Chinese hamster ovary $(\mathrm{CHO})$ cell line. Although alternative hosts, such as yeast, Escherichia coli and plants, have been proposed to have benefits of rapid genetic engineering and production, major deficiencies preclude them from enabling a rapid response to a pandemic outbreak. The established cGMP production infrastructure cannot support large clinical trials and post-licensure demand. For such hosts, the lack of clinical experience presents a substantive patient safety risk arising from the potential impacts of rare $\mathrm{mAb}$ post-translational modifications or variants, including unusual glycans, host cell proteins, and so forth. In contrast, the low risk profile of $\mathrm{mAbs}$ produced by $\mathrm{CHO}$ cell lines has been established and supports a rapid development model based on platform development, manufacturing technology and infrastructure.

Several companies have developed cell lines using targeted integration of $\mathrm{mAb}$ expression vectors ${ }^{6,7}$. These cell lines provide more consistent expression through integration of low copy numbers in highly active transcriptional hotspots. This consistency can reduce the time for screening cell pools or clones leading to a phase 1 cell line. By not assessing multiple pools of transfectants, generating interim cell banks, and assessing productivity of pools as part of the routine cell line development used for decades with random integration, savings of several months could be gained between transfection and cloning. (Although targeted integration is a critical advance, it is possible that an optimized random integration technology may also produce a high percentage of transfectants with suitable productivity.)

Moving directly from the stable transfectant pool to cloning is becoming a standard practice today. Until recently, an intermediate stage of expansion generation of several pools of transfectants and subsequent screening - was used to increase the probability of finding a 


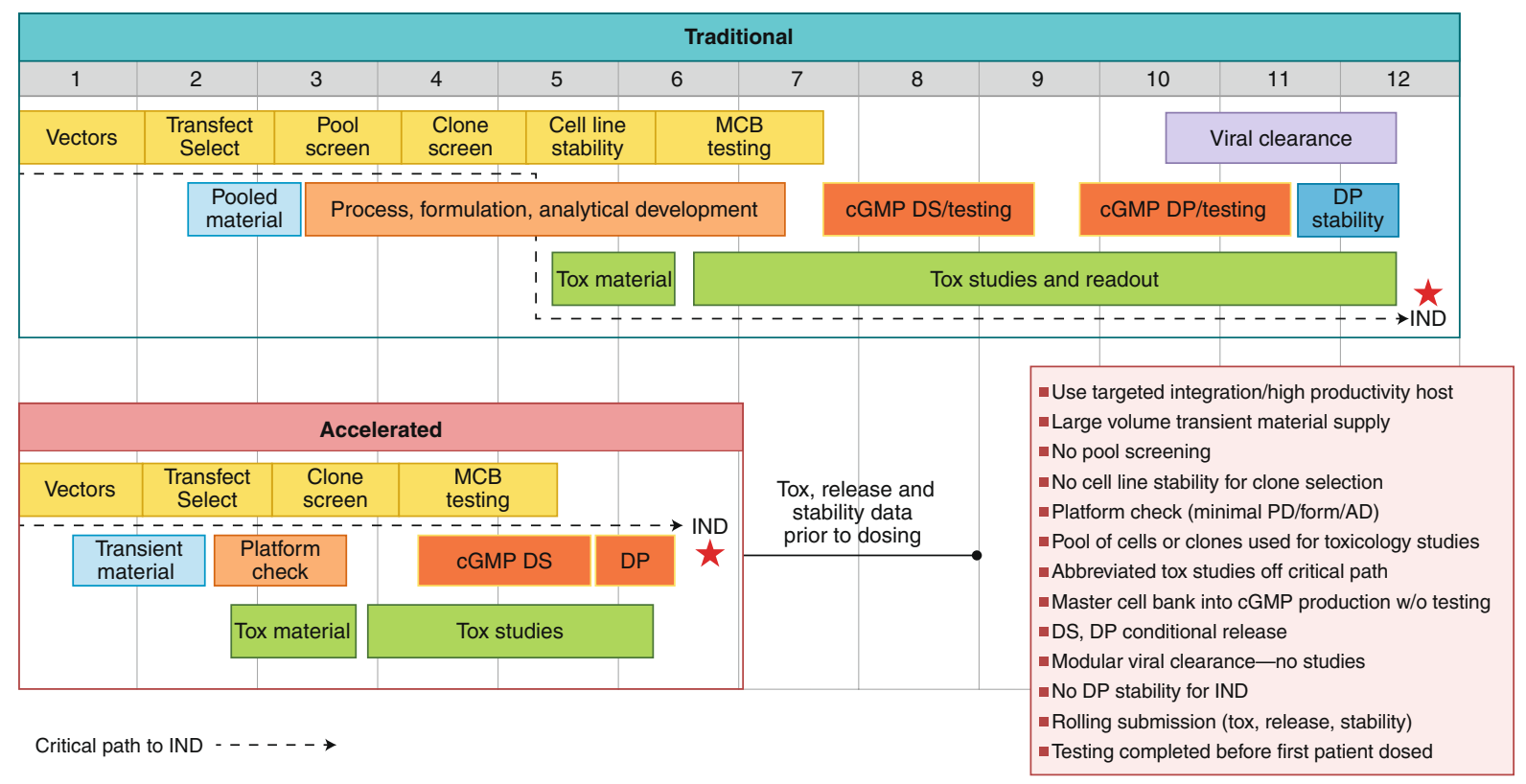

Fig. 1 | Accelerated phase 1 CMC mAb timeline for a pandemic. The timeline to phase 1 clinical studies using mAb therapeutics for pandemic outbreaks can be substantially accelerated without heightened product safety risks as compared with current practice. Tox, toxicology; MCB, master cell bank; DS, drug substance; $\mathrm{DP}$, drug product; PD, process development; form, formulation; $\mathrm{AD}$, analytical development.

high-producing line, but this takes many weeks, including the typical 2-week production culture screen followed by analysis of product quality. If instead one moves directly to cloning from a pool of transfectants with consistent productivity, the final clone screening step could be conducted much earlier. Another few weeks may also be saved by conducting a single round of cloning using fluorescence-activated cell sorting (FACS) or limiting dilution, with supporting imaging to establish the clonal derivation of the resulting cell line, rather than performing two rounds of limiting dilution ${ }^{8,9}$.

Finally, multiple candidate clones can be screened with very small bioreactors using small-volume tubes or ambr 15 bioreactors of $15 \mathrm{~mL}$ volume ${ }^{10}$, which could save roughly 5 days instead of screening using 5-liter bioreactors.

In aggregate, these new technologies and approaches could save 2 months in the timeline from lead identification to establishment of a clonally derived cell line suitable for phase 1 production (Fig. 1). If toxicology studies are shortened, chemistry, manufacturing and control (CMC) activities may comprise the critical path to the IND filing.

Process and formulation development In parallel with cell line development, transient expression cultures produce material to support downstream process, formulation and analytical development.
Large-scale transient cultures ( $\geq 100$ liters) generate many grams of product in a single batch $^{11}$. The availability of this feedstock weeks earlier than material from clonal cell lines accelerates the timeline to cGMP production, informing the final process definition and drug product formulation.

\section{The fastest process \\ development strategy for clinical studies precludes optimization or evaluation of process performance at \\ pilot scale.}

By selecting an IgG1 mAb, one can leverage experience with platform processes and production facilities. High-throughput screening of platform polishing chromatographic steps uses very little material and is highly predictive of process performance ${ }^{12}$. These studies can be conducted before the final clone selection, with little risk of an impact on the downstream process.

Restricting the use of raw materials to those that have already been procured and tested and are available in the cGMP warehouse enables the fastest timeline to production. Although this is a constraint on the choice of chromatography resins, late-stage development provides an opportunity to optimize the process and resin selection for higher loadings, reduction from two to one polishing chromatography steps $^{13}$, and further process intensification.

The fastest process development strategy for clinical studies precludes optimization or evaluation of process performance at pilot scale. Following cell line selection, one would proceed directly to cGMP production. For the sake of speed, you 'take what you get' from the platform process and save months in the cycles of small-scale and pilot studies normally used to assess the clinical process performance and consistency. This accepts a risk that the cGMP manufacturing or product will be unacceptable. In a pandemic outbreak, this an acceptable risk that would not compromise patient safety as product quality would be tested by cGMP quality control labs, and if judged unsuitable, the material would not be used. Even in the event that product quality was not in the range that would be appropriate for commercial production (for example, elevated high-molecular-weight species or residual host cell proteins), there is a reasonable expectation that late-stage development would improve the product quality profile and this material would be suitable for early clinical studies.

\section{Portability, dosage and formulation}

The production design basis of most therapeutic mAbs is quite similar ${ }^{14}$. Using a platform process for phase 1 production ensures portability to other manufacturing facilities. If there is a need to quickly 
produce hundreds of thousands of doses (100 kg or more), this common design basis would be key to accomplishing this objective.

The drug product configuration must accommodate the appropriate dose and route of administration. If the dose is large for infectious diseases ( $>500$ milligrams or more), the volume limits of subcutaneous administration will be exceeded for conventional injections, and intramuscular or intravenous administration routes would be used. High-concentration liquid formulations ( $\geq 150$ milligrams per milliliter) would be advantageous, but given the limited development time available more conservative concentrations of 50-75 milligrams per milliliter and frozen or lyophilized drug product storage should be considered. A lyophilizate has drawbacks of the time required for processing (3 or more days longer than liquid) plus the greater risk of running a cGMP batch without a pilot run if materials or time were limiting.

Thus, the initial clinical entry product profile would be a liquid drug product at a moderate concentration, stored cold or frozen. The preformulation screen would evaluate only proven formulations and excipients. The container-closure materials should likewise be stock items. Establishing long-term (multi-year) stability for early clinical material would not be required; resupply with new batches of drug substance and/or rapid consumption of drug product in the clinic combine to limit any risk of poor stability of the formulated drug product.

\section{Toxicology}

Many companies have used pools of clones or transfection pools to produce material for preclinical toxicology studies ${ }^{15-19}$. The suitability of this material is then established through product release and extended characterization test comparisons to clinical material. This strategy accelerates the start of toxicology studies. For conventional programs, the start of the toxicology study is on the critical path to filing an IND, but a short study could shift the critical path back to $\mathrm{CMC}$, including production of clinical trial material.

In the case of a pandemic outbreak, one can evaluate whether phase 1 toxicology studies could be eliminated altogether or conducted in parallel with IND review and product distribution. Tissue cross-reactivity studies are vital to ascertain whether the $\mathrm{mAb}$ binds to epitopes other than the targeted pathogen epitope. For the situation in which the $\mathrm{mAb}$ recognizes a viral antigen and was also isolated from a human, does this reduce the risk of off-target binding to the point where minimal or no toxicology studies are required before human clinical testing?

\section{cGMP production and analytics}

One could move the master cell bank into production immediately without completing the full panel of testing. Some assays have long test periods (up to 40 days), and passing results are a requirement for facility entry. Using an unreleased cell bank in a cGMP facility is a business risk but not a product safety risk. If the cell bank were to fail any release tests (a very low probability given current practices and controls), the impact to the facility would be assessed and may delay the start-up of subsequent campaigns. Yet the release testing of the cell bank would be completed before any product would be distributed, thus precluding any safety risk to patients. Another option that would accelerate the cGMP production would be to derive the seed culture from the expanded culture at the time the master cell bank is deposited by using half of the expanded culture for banking and the other half to initiate cGMP production. Although this first batch would therefore not be derived from a master cell bank vial, approximately 1 week could be saved.

\section{Colocation of the drug substance and drug product manufacturing and joint operation by the same company would provide time savings associated with shipping of drug substance between sites or companies.}

In an emergency scenario where every day counts, one could use the minimum production scale for the first batches (for example, 200 liters versus 2,000 liters) and thus save 3-4 days in the inoculum stage of production. Parallel production trains could provide both small-volume batches quickly and larger-volume batches soon afterwards.

The application of single-use bioreactors enables rapid entry into clinical cGMP production facility, as well as accelerated changeover between batches. Currently, 1,000-2,000 liter single-use bioreactors are commonly used, but recently 4,000 and 6,000 liter bioreactors have been launched. The production capacity from these large bioreactors could supply many thousands of doses and could also build up a launch supply of drug substance.

Colocation of the drug substance and drug product manufacturing and joint operation by the same company would provide time savings associated with shipping of drug substance between sites or companies. For drug product manufacturing, disposable filling lines and robotic fill-finish in fully contained isolators may also speed up production in some scenarios where product changeover of conventional filling lines takes a few days.

There is a scenario wherein cGMP production directly from the transfectant pool could be performed. The time savings comes from not performing a cloning step and subsequent expansion to generate a master cell bank. The use of non-clonally derived cells is not an accepted practice today, but in the case of a pandemic outbreak it should be considered. This would be a faster means of producing cGMP material (as much as 6 weeks), but the savings may not warrant the associated regulatory and future product comparability complexities as the production process transitions over to a clonally derived cell bank for subsequent clinical trials.

For product testing and release, platform analytics for IgG1 mAbs enable rapid cGMP product release. Release specifications could be established using typical ranges for mAbs in phase 1 studies without the need for product-specific limits unless warranted ${ }^{20}$. A more time-consuming test to develop, such as the antigen-binding enzyme-linked immunosorbent assay (ELISA), may be qualified during IND review or performed as 'confirmatory' after the IND is filed.

\section{Quality and regulatory considerations}

Similarly to the testing of a master cell bank in parallel with cGMP production, drug substance and drug product could be conditionally released for further processing. Their release testing would be performed while the subsequent processing was underway (for the drug product, this would be labeling and packaging). Critical tests ensuring safety, such as sterility, identification and endotoxin, would be completed before distribution, but characterization tests could lag behind final release.

Another activity that could be on the critical path is viral clearance studies. Platform process steps with a sufficient performance database would enable modular claims of viral clearance without the need to conduct product-specific studies $^{21}$. These apply to steps such as low-pH inactivation, virus filtration and chromatography.

A rolling IND submission enables the fastest clinical start if non-clinical data, product stability or characterization data would hold up the IND filing. Early contacts 
between the sponsor and regulatory authorities would enable the development of a strategy for joint review, discussion of risk mitigation options and approval of clinical development plans. Recently issued guidance from the US Food and Drug Administration on Emergency Use Authorization also opens opportunities that may permit the agency to waive otherwise applicable cGMP requirements ${ }^{22}$.

\section{Clinical supply chain}

The initial drug product expiry could be established on the basis of platform knowledge and enable the earliest clinical start. Given the speed to cGMP production, assessing the stability of the toxicology lot may be of little benefit. The mAb stability profile under accelerated and stressed temperatures could be compared with those of other mAbs and would determine whether the product had any unusual degradation pathways or kinetics. Product expiry dating would be extended by extrapolation based on real-time data.

The initial clinical production capacity could sustain reasonably large trials to confirm initial safety and efficacy results, depending on the dose. A 2,000-liter batch with a titer of 3-4 grams per liter would yield 3,000-5,000 1-gram doses. For larger single-use bioreactors, or when multiple 2,000-liter bioreactor harvests are pooled into a single batch, the downstream process capacity may become limiting. Strategies such as sub-batching and unit operation cycling could relieve this limitation.

If the product shows efficacy in early clinical studies, a rapid increase in capacity would be needed. Process transfer to more manufacturing sites and/or scale-up of the phase 1 process would be the fastest ways to achieve this objective.

\section{Later stages of clinical development and licensure}

If the early stages of clinical development show promising product safety and efficacy, then the product would progress to later clinical stages of clinical development and potential product licensure. The opportunities to accelerate product licensure are manifold, and these details are beyond the scope of this paper, but some considerations are described here.

If the phase 1 cell line productivity does not enable sufficient commercial supply, a new cell line could be introduced in later clinical studies. This may be more likely than current $\mathrm{mAb}$ development strategies in which a single cycle of cell development is preferred; this objective drives the longer timelines of cell line development that seek to identify clones with high productivity and stability and low levels of product sequence variants. This 'next generation' cell line development would enable time for assessing technologies such as gene amplification, supertransfection, evaluation of thousands of clones and high-throughput analytics.

The manufacturing process could also be optimized. The objectives could include increased volumetric productivity (increasing titer and shortening the production culture duration ${ }^{23}$ ) and intensifying the downstream process (using a single chromatography polishing step $^{24}$, minimizing solution volumes and enabling in-line buffer dilution).

\section{The portability of the commercial process to enable production at 15,000- 25,000 liters is an important requirement of a process that must be capable of supplying millions of doses in a short time or building up a stockpile of product.}

Before the final definition of the commercial process, it could be advantageous to conduct a rigorous drug substance facility fit analysis with multiple contract manufacturing organizations (CMOs) and potential innovator company partners. The portability of the commercial process to enable production at $15,000-$ 25,000 liters is an important requirement of a process that must be capable of supplying millions of doses in a short time or building up a stockpile of product. Multiple drug substance and drug product manufacturers may be required. A consortium of two or more companies sharing production capacity was proposed ${ }^{25}$ in 2006 and may be necessary to supply the large volume of product required.

The drug product could also be changed from the configuration used in phase 1 trials. An improved liquid formulation enabling cold storage would be valuable for a stockpile. Alternatively, container-closure components that enable storage as a frozen drug product could markedly extend the shelf life of the drug product.

Production of the phase 3 drug substance and drug product lots provides an opportunity for establishing a dataset suitable for the process validation package of the license application. Appropriate sampling, testing and data analysis of the phase 3 lots could preclude the need for a separate qualification campaign executed months later, which is the typical practice today, accelerating the license application ${ }^{26}$. This would require that there be no significant process changes after the phase 3 campaign. After licensure, continuous process verification would be employed to manage the early years of commercial production ${ }^{27}$.

\section{Synergistic effects of an integrated timeline on speed to clinic}

If speed to a phase 1 trial for $\mathrm{mAb}$ therapies has been a focus of the biopharmaceutical industry for many years, what is new about this approach for a pandemic outbreak? Benchmarking with innovator companies and CMOs suggests the current best-in-class timeline from lead identification to IND timeline is $10-12$ months.

The first aspect that is new in the pandemic approach is that several strategies and new technologies are combined into a single program. Companies have worked on many of these innovations, but not all have been assembled together. Targeted integration is a major advance and is only a few years old. The use of 15-milliliter bioreactors for clone screening is relatively new. Using a pool of cells for production of material for toxicology studies is a recent development. Using a cell line (or even a pool) for cGMP production as fast as possible without satisfying the performance requirements of a commercial cell line enables substantial time savings. Including a single round of cloning plus capturing an image of the single cell in the well, large-scale transient cultures supporting process and formulation development are recent advances, as are single-use drug substance and drug-product manufacturing. The combination of several time savings adds up to a major improvement in the estimate of the overall timeline duration. These are represented in concert in Fig. 1.

Second, some approaches to regulatory and quality compliance that reflect the 'need for speed' during a pandemic outbreak also reduce development times. Highly abbreviated toxicology studies would likely shift the critical path back to CMC. Accepting business (but not product safety) risks - including cGMP processing at risk before cell bank testing is complete, conducting minimal product quality release testing, employing modular viral clearance, and conducting a rolling IND filing would likely also contribute to accelerating the overall timeline.

The combination of these technical and regulatory/compliance advances may be synergistic, resulting in a substantial acceleration to introducing a mAb into the 


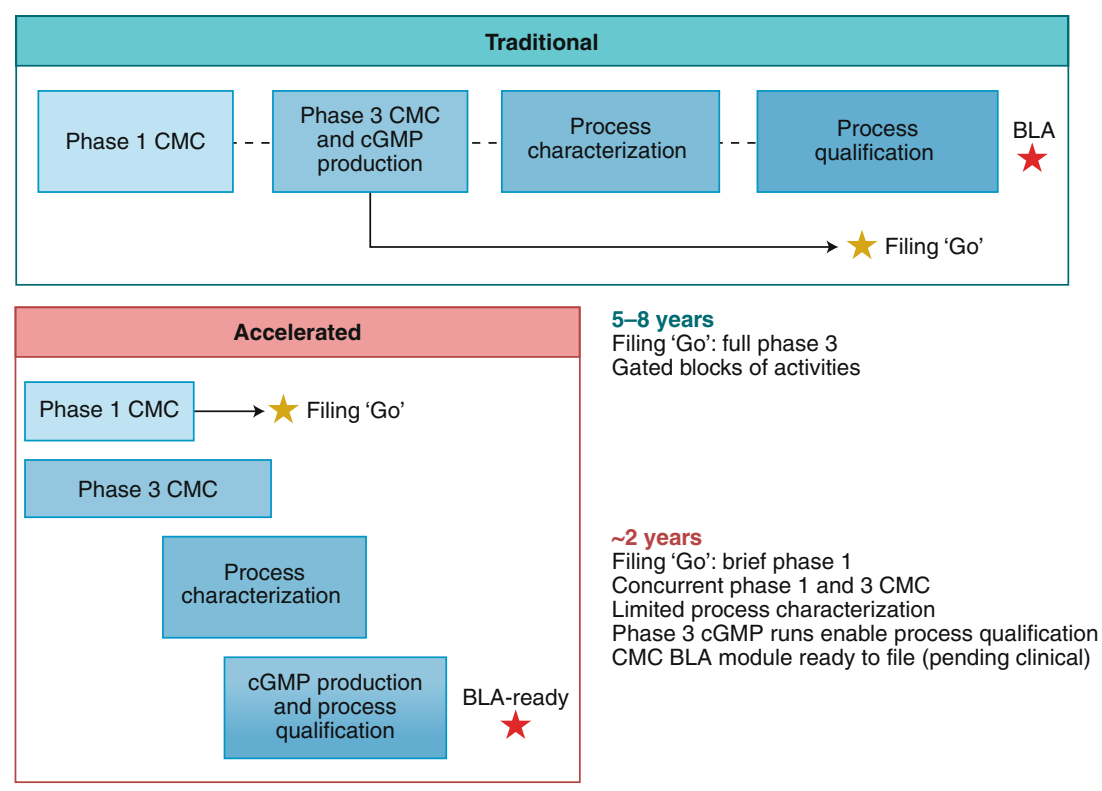

Fig. 2 | mAb product development for pandemics (mAb lead identification to BLA). The fastest path to licensure for therapeutic $\mathrm{mAbs}$ treating pandemic outbreaks requires accepting business risks and preinvestment, but without major product quality, supply and regulatory risks.

clinic. Yet there should be no increased patient safety risk for an established therapeutic modality such as an IgG1 $\mathrm{mAb}$ produced with established platform processing, formulations and testing.

The global regulatory landscape may influence the development strategy, as the variation in health authorities' experiences and risk tolerances could influence the regional speed to clinic and development plans. Current regulatory harmonization approaches are not configured to enable coordinated clinical testing.

\section{Implications}

During a pandemic outbreak every day counts, and several examples described above could combine to reduce the critical path to the first clinical trials. Although yet to be proven, the approach described above to supplying phase 1 studies could reduce today's best-in-class timeline for lead $\mathrm{mAb}$ identification to IND from 10-12 months to 5-6 months.

Looking beyond the fastest timeline to phase 1 clinical trials, the subsequent stages of pivotal clinical trial testing and product launch come into focus. By leveraging the process and product platform knowledge for IgG1 products, as well as the established manufacturing infrastructure at CMOs and innovator companies, it should be possible to launch a $\mathrm{mAb}$ product in just a few years. Would a timeline of 2 years from identification of the lead $\mathrm{mAb}$ to a having the CMC module of a Biologics
License Application (BLA) filing be possible, given an IgG1's safety profile, rapid clinical evaluation, at-risk business investment, and streamlined CMC strategies for the phase 1 and licensure (Fig. 2)? Post-licensure supply and/or stockpiling could exploit highly efficient platform production and an established network of manufacturing partners to support the demand for a product that could be unprecedented in production scale ( $>10$ tons per year, equivalent to 10 million or more doses).

\section{The combination of}

approaches described here

in the context of a pandemic disease outbreak could reduce the time from the current standard of mAb lead identification from an already rapid IND of 10-12 months to potentially as little as

\section{5-6 months.}

Large pharmaceutical companies with an established technology base and production facilities may be well positioned to implement these strategies. Smaller companies would need to rely on partners in the contract manufacturing sector that can move quickly through the process development and manufacturing stages, as well as have sufficient throughput to immediately bring one or more new mAbs into their pipeline.

\section{Pre-established partnerships and pandemic preparedness}

Another aspect that deserves consideration is the establishment of collaborations between discovery companies and CMOs or innovator companies with manufacturing capacity in anticipation of the need to execute the development strategy described here. These collaborations could take the form of material transfer, supply, quality and legal agreements, as well as financial contracts.

Even when the product discovery and $\mathrm{CMC}$ assets are managed by the same company, the response to a pandemic outbreak is likely to be a difficult challenge and would require changes to existing governance and decision-making systems. The large effort required for business and legal departments to execute the necessary agreements could delay the initiation of the first stages of CMC development and hence the critical path to clinical trial initiation. In this respect, open material transfer agreements are starting to be considered for tech transfer in the academic setting.

Different partners for clinical and commercial phases may be required. The ideal partner for rapid phase 1 production may not be the same for commercialization. The manufacturing partner could stockpile some raw materials in advance (cell culture media, downstream resins and filters, drug product components) to avoid procurement becoming rate-limiting to production. It may also be necessary to procure certain equipment in advance (for example, large-volume drug substance storage containers, dedicated drug product filling needles, or tanks).

The production capacity needed to produce sufficient material to support early- and late-stage clinical trials would not typically be a limitation provided the manufacturing facilities are available. The subsequent steps required for product launch (process scale-up, process transfers, and the securing of capacity at a much larger facility) could, however, become rate-limiting to establishing a regular supply of a large number of doses for distribution.

\section{Conclusions}

The fastest timeline from lead $\mathrm{mAb}$ identification to phase 1 studies is an important goal for all companies and the patients they treat. Many companies developing therapeutic mAbs have worked tirelessly to refine their technology and strategies to enable rapid clinical evaluation, 
converging on a typical timeline for initial clinical studies. The combination of approaches described here in the context of a pandemic disease outbreak could reduce the time from the current standard of $\mathrm{mAb}$ lead identification from an already rapid IND of 10-12 months to potentially as little as 5-6 months.

It will not escape the reader's notice that the combination of these acceleration strategies for addressing a pandemic outbreak could potentially be applied to clinical development for all other mAbs, for all other indications.

\section{Editor's note: This article has been peer-reviewed.}

Brian Kelley (iD $₫$

Vir Biotechnology, San Francisco, CA, USA.

$凶_{e-m a i l: b k e l l e y @ v i r . b i o}$

Published online: 21 April 2020

https://doi.org/10.1038/s41587-020-0512-5

References

1. Zhu, Z. et al. Proc. Natl Acad. Sci. USA 104, 12123-12128 (2007).
2. Corti, D et al Science 333, 850-856 (2011).

3. Stettler, K. et al. Science 353, 823-826 (2016).

4. Lu, R.-M. et al. J. Biomed. Sci. 27, 1-30 (2020).

5. The Antibody Society. Antibody therapeutics approved or in regulatory review in the EU or US. https://www.antibodysociety. org/resources/approved-antibodies/ (2020).

6. Rajendra, Y. et al. Biotechnol. Prog. 33, 1436-1448 (2017)

7. Hamaker, N. K. \& Lee, K. H. Curr. Opin. Chem. Eng. 22, 152-160 (2018)

8. Shaw, D. et al. Biotechnol. Prog. 34, 584-592 (2018)

9. Zingaro, K. et al. PDA J. Pharm. Sci. Technol. 72, 438-450 (2018).

10. Sandner, V., Pybus, L. P., McCreath, G. \& Glassey, J. Biotechnol. J. 14, 1700766 (2019).

11. Gutiérrez-Granados, S., Cervera, L., Kamen, A. A. \& Gòdia, F Crit. Rev. Biotechnol. 38, 918-940 (2018).

12. Coffman, J. L., Kramarczyk, J. F. \& Kelley, B. D. Biotechnol. Bioeng. 100, 605-618 (2008)

13. Kelley, B. D. et al. Biotechnol. Bioeng. 101, 553-566 (2008).

14. Kelley, B. MAbs 1, 443-452 (2009).

15. Hu, Z. et al. Biotechnol. Prog. 33, 1449-1455 (2017).

6. Munro, T. P. et al. Biotechnol. Prog. 33, 1476-1482 (2017).

17. Fan, L. et al. Biotechnol. Prog. 33, 1456-1462 (2017)

18. Wright, C., Alves, C., Kshirsagar, R., Pieracci, J. \& Estes, S. Biotechnol. Prog. 33, 1468-1475 (2017)

19. Scarcelli, J. J., Shang, T. Q., Iskra, T., Allen, M. J. \& Zhang, L. Biotechnol. Prog. 33, 1463-1467 (2017)

20. Kretsinger, J. et al. J. Pharm. Sci. 108, 1442-1452 (2019).

21. Stuckey, J. et al. Biotechnol. Prog. 30, 79-85 (2014).

22. U. S. Food and Drug Administration. Emergency use authorization of medical products and related authorities. https://www.fda.gov/regulatory-information/search-fdaguidance-documents/emergency-use-authorization-medicalproducts-and-related-authorities (2017).
23. Yang, W. C. et al. Perfusion seed cultures improve biopharmaceutical fed-batch production capacity and product quality. Biotechnol. Prog. 30, 616-625 (2014).

24. Kelley, B. Biotechnol. Prog. 23, 995-1008 (2007).

25. Kamarck, M. E. Nat. Biotechnol. 24, 503-505 (2006).

26. Eschelbach, J. Registration Enabling Campaign for Accelerated Development: a PPQ strategy with early investment to enable acceleration for a promising monoclonal antibody. https://cdn. ymaws.com/www.casss.org/resource/resmgr/cmc_euro/ CMC_Europe_2017_Program_vl.pdf (2017).

27. Boyer, M., Gampfer, J., Zamamiri, A. \& Payne, R. PDA J. Pharm. Sci. Technol. 70, 282-292 (2016).

Acknowledgements

The author thanks employees of WuXi Biologics including Chris Chen, Sherry Gu, Jiexing Cai and Ying $\mathrm{Hu}$ for close collaborations and discussions regarding strategies for accelerating development timelines for initial clinical trials. Colleagues at Vir Biotechnology including Kristen Koterba Douglas, Davide Corti, Lori Houle, Jay Howlett, Irene Pleasure, Todd Renshaw, Lynne Krummen and Michael Kamarck provided invaluable input to the concepts and strategies described in this manuscript. A broad group of biopharmaceutical technical development leaders also contributed during many years of benchmarking.

\section{Competing interests}

The author is employed by Vir Biotechnology, which is developing monoclonal antibodies for the treatment of pandemic diseases, such as COVID-19 and influenza. 